\title{
Polymorphism of IL37 gene as a protective factor for autoimmune thyroid disease
}

\author{
Ni Yan, Shuai Meng, Rong-Hua Song, Qiu Qin, Xuan Wang, Qiuming Yao, Yanfei Jiang, \\ Wenjuan Jiang, Liangfeng Shi, Jian $X u$ and Jinan Zhang
}

Department of Endocrinology, Jinshan Hospital of Fudan University, Number 1508 Longhang Road, Jinshan District, Shanghai 201508, China
Correspondence should be addressed to J Zhang Email zhangjinan@hotmail.com

\begin{abstract}
Autoimmune thyroid disease (AITD) comprises Graves' disease (GD) and Hashimoto's

thyroiditis (HT). IL37 has been recently proved to be a natural suppressor for innate immunity and acquired immunity. Therefore, this study was conducted to identify the association of IL37 genetic polymorphisms with AITD in Chinese Han population. Polymorphisms of rs3811046/rs3811047/rs2723176/rs272186 in the IL37 gene were assessed in a case-control study comprising $701 \mathrm{GD}$ patients, $301 \mathrm{HT}$ patients and 939 controls. Genetic variants were genotyped by multiplex polymerase chain reaction and ligase detection reaction. The frequencies of the minor allele $A$ of rs2723176 and A of rs2723186 were significantly lower in the GD patients than in the controls $(P=0.014, \mathrm{OR}=0.774 ; P=0.014, \mathrm{OR}=0.777)$. After gender stratification, the rs3811046 G allele and the rs3811047/rs2723186 A allele were both significantly associated with a decreased risk of GD in female patients $(P=0.030, \mathrm{OR}=0.777$; $P=0.023, \mathrm{OR}=0.774 ; P=0.029$, OR=0.761). However, none of the four single nucleotide polymorphisms of IL37 gene showed any significant association with HT. Moreover, haplotype analysis revealed the GCG haplotype conferred increased risk for GD as a whole and in female $G D$ patients $(O R=1.213 ; O R=1.320)$. The $A C G$ haplotype was associated with an increased risk of $H T$ as a whole $(O R=1.567)$ and in male $G D$ patients $(O R=1.820)$. In contrast, the AAA haplotype showed a protective role for $G D$ as a whole $(O R=0.760)$ and in female GD patients $(O R=0.765)$. Our study strongly supports that the IL37 gene variants are associated with the susceptibility to AITD.
\end{abstract} Key Words

- IL37

- IL1F7

- single nucleotide polymorphisms (SNPs)

- autoimmune thyroid diseases (AITDs)

- Graves' disease (GD)

- Hashimoto's thyroiditis (HT)

Journal of Molecular

Endocrinology

(2015) 55, 209-218

\section{Introduction}

Autoimmune thyroid diseases (AITDs), typically including Graves' disease (GD) and Hashimoto's thyroiditis (HT), are the most common organ-specific autoimmune disorders, affecting up to $5 \%$ of the overall population (Tomer \& Huber 2009). GD and HT represent the major portion of clinical expressions of thyroid dysfunction but differ in their clinical presentations as well as pathophysiology. HT is a $\mathrm{T}$ cell-mediated organ-specific autoimmune disease characterized by lymphocytic infiltration that leads to thyroid cells loss, clinically manifested as hypothyroidism (Antonelli et al. 2015). In contrast, GD is characterized by hyperthyroidism due to overproduction of thyroid hormone induced by specific auto-antibodies against thyrotropin receptor (TSHR) (Morshed et al. 2012).

The etiology of GD and HT involves common and unique pathways. Both diseases carry thyroid $\mathrm{T}$ cells that

Published by Bioscientifica Ltd 
escape the immune tolerance process and infiltrate the thyroid. However, distinct pathways lead thyroid-reactive $\mathrm{T}$ cells to cause either apoptosis of thyroid cells in HT or their overgrowth in GD (Tomer 2010). The current paradigm is that AITDs are multifactorial diseases. Several factors, including genetic, hormonal, environmental and nutritional elements, are involved in the initiation and/or development of AITDs. However, the pathophysiologic changes seen in AITDs are mediated by inflammatory cytokines (Ganesh et al. 2011). Cytokines are crucial regulators to promote or suppress inflammation. Single nucleotide polymorphisms (SNPs) of inflammatory cytokines may change the transcription rate and consequently the production of cytokines (Dominici et al. 2002, Wen et al. 2006, Prasad et al. 2010), and thus may modify individual susceptibility to AITDs. Various cytokines and respective genetic polymorphisms have been reported to be associated with AITD, but the results are inconsistent (Chen et al. 2005, Shiau et al. 2006, Anvari et al. 2010, Liu et al. 2010, Yan et al. 2012).

The interleukin 1 gene family (IL1F) cluster is located on chromosome $2 \mathrm{q} 13$, within a $36 \mathrm{~kb}$ region, including IL1A, IL1B, IL1F7, IL1F9, IL1F6, IL1F8, IL1F5, IL1F10 and IL1RN (Nicklin et al. 2002). The IL1 molecule has extensive biological effects, among which is its ability to partly or entirely activate and enhance the function of mononuclear cells, neutrophils and phagocytes and promote them to release inflammatory mediators; it also mediates immune response and inflammation reactions. A genomewide scan has identified that a region including IL1 family gene cluster is linked to ankylosing spondylitis (AS) susceptibility (Miceli-Richard et al. 2004, Timms et al. 2004,). This linkage finding was later confirmed by one family-based association study in western Canadian family population (Zhang et al. 2004). IL37 was formerly termed as an IL1 family member 7 (IL1F7). Studies have shown that the biological properties of IL37 are mainly those of downregulating the production of pro-inflammatory cytokines and protecting mice from inflammatory and autoimmune diseases, as assessed in models where human IL37 is expressed in mice (McNamee et al. 2011, Sakai et al. 2012).

Recently, clinical studies have demonstrated that the IL37 gene SNP rs3811047 G>A is related to the susceptibility to autoimmune diseases, such as ankylosing spondylitis (AS) (Pan et al. 2010), disease activity of rheumatoid arthritis (RA) (Pei et al. 2012), and gastric cardiac adenocarcinoma (Wang et al. 2014).

In our unpublished study, we found that the mRNA level of IL37 was significantly high in peripheral blood mononuclear cells of GD patients. To our knowledge, there has been no study to report the relationship between SNPs of IL37 and AITD patients so far. Therefore, the aim of the present study was to analyze the genetic association of the four SNPs of IL37 (rs3811046/rs3811047/rs2723176/ rs2723186/) with AITD in a large cohort of Chinese Han population. Furthermore, we analyzed the association between genotypes and AITD clinical phenotypes.

\section{Materials and methods}

\section{Ethics statement}

The research protocol was approved by the Ethics Committee of Jinshan Hospital of Fudan University (2014-05-01; 2015-01-01). Written informed consents were obtained from all participants and/or their parents.

\section{Study population}

The study population $(n=1999)$ consisted of 1061 unrelated Chinese Han AITD patients, including 701 patients with GD and 360 patients with HT, and 938 unrelated, ethnically and geographically matched healthy controls. All AITD patients were enrolled from the Outpatient Department and Inpatient Department of Endocrinology of Jinshan Hospital of Fudan University. The healthy controls were recruited from the Health Check-Up Center of the same hospital. The subjects were all of selfreported Chinese Han ethnicity.

\section{Clinical assessment}

Inclusion criteria: AITDs patients were diagnosed as described in our published papers (Yan et al. 2014). The diagnosis of GD was based on the presence of clinical and laboratory biochemical hyperthyroidism with diffuse goiter, decreased TSH value, increased levels of free thyroid hormones and supported by the positive anti-thyroid stimulating hormone receptor antibody (TRAb) and/or anti-thyroid peroxidase antibody (TPO-Ab) and/or antithyroglobulin (Tg-Ab) and/or exophthalmos. HT was diagnosed based on the presence of an enlarged thyroid and either TPO-Ab or Tg-Ab, with or without documented clinical and biochemical hypothyroidism. For the suspicious cases of HT, diagnoses were confirmed by fine needle aspiration biopsies (FNAB).

Exclusion criteria: all healthy controls had to exhibit no evidence of goiter, or TPO-Ab/Tg-Ab nor any personal or family history of thyroid diseases or any other autoimmune diseases.

Published by Bioscientifica Ltd. 
The clinical phenotypes of AITD patients included: i) adult- or childhood-onset AITDs, according to the age at the time of disease onset ( $\leq 18$ years vs $\geq 19$ years); ii) presence or absence of ophthalmopathy in the GD group (defined as a distinctive disorder characterized by inflammation and swelling of the extraocular muscles, eyelid retraction, periorbital edema, episcleral vascular injection, conjunctive swelling and proptosis); iii) presence or absence of thyroid dysfunction in HT group: euthyroid status or hypothyroidism; and iv) presence or absence of AITD family history.

\section{Peripheral blood DNA extraction}

Peripheral venous blood of $2 \mathrm{ml}$ was collected from all the study participants. Genomic DNA was extracted from peripheral blood cells using the Relax Gene Blood DNA System (Tiangen Biotech Co., Ltd., Beijing, China), according to the manufacturer's guidelines. The concentration and purity of each DNA sample were measured by Nano Drop 2000 Spectro-photometer (Thermo Scientific Company, Waltham, MA, USA).

\section{SNP selection and genotyping}

Four SNPs (rs3811046/rs3811047/rs2723176/rs2723186) of IL37 gene were selected from the Hapmap CHB data (http://hapmap.ncbi.nlm.nih.gov/) using Haploview Software 4.2 (Barrett et al. 2005). According to previously published literature, polymorphisms of these locis may also be associated with the susceptibility to AS or RA (Pan et al. 2010, Pei et al. 2012). All the tag SNPs met the following criteria: minor allele frequency $(\mathrm{MAF})>0.05$, Hardy-Weinberg equilibrium (HWE) with $P>0.001$ and logarithm of odds (LOD) $>3$.0. The minor allele frequency of the four SNPs among Chinese Han in Beijing, China (CHB) was as follows: rs3811046 $M_{\mathrm{G}}=0.146$, rs3811047 $M_{\mathrm{A}}=0.146, \mathrm{rs} 2723186 M_{\mathrm{A}}=0.102, \mathrm{rs} 2723176 M_{\mathrm{A}}=0.103$.

The genotyping of four SNPs of IL37 was conducted by Shanghai Biowing Applied Biotechnology Company (http://www.biowing.com.cn/) using ligase detection reactions (LDR) platform (Yan et al. 2014). The target DNA sequences were amplified using multiplex polymerase chain reaction (PCR) method with specific primer sequences as follows: rs3811046 forward: 5'-GAGGCCTTACTTGTGTGAAC-3'; reverse: 5'-AACCTCACTGCGTCTGACTG-3'; rs3811047 forward: 5'-GAGGCCTTACTTGTGTGAAC-3'; reverse: 5'-AACCTCACTGCGTCTGACTG-3'; rs2723176 forward: 5'-ACAGACCTCCTGCCTCAGAA-3'; reverse: 5'-AAGGTGCTGGCAGTTGTTTT-3'; rs2723186 forward: 5'-AAGGAGGCAGACACTTAGAC-3'; reverse: 5'-TTGACTCTGTGGTCCACTTC-3'.

Extracted DNA was amplified by PCR using the Gene Amp PCR System 9600 (Perkin Elmer, Waltham, MA, USA). The amplification procedure consisted of initial denaturation at $95^{\circ} \mathrm{C}$ for $2 \mathrm{~min}$, denaturation at $94^{\circ} \mathrm{C}$ for $90 \mathrm{~s}$, annealing at $65^{\circ} \mathrm{C}$ for $30 \mathrm{~s}$, extension at $65^{\circ} \mathrm{C}$ for $30 \mathrm{~s}$ with 40 cycles and final extension at $65^{\circ} \mathrm{C}$ for $10 \mathrm{~min}$. After the reaction, $2 \mu \mathrm{l}$ of each product was run in a $3.0 \%$ agarose gel to determine whether the reaction had been successful. After completion of the amplification, the ligation detection reaction for each subject was carried out in a final volume of $10 \mu$ c containing $1 \mu \mathrm{l}$ of buffer $(1 \times), 1 \mu$ of probe $\operatorname{mix}(2 \mathrm{pmol} / \mu \mathrm{l}), 4 \mu \mathrm{l}$ of Multi-PCR product (100 ng/ $\mu \mathrm{l}), 4 \mu \mathrm{l}$ of $\mathrm{H}_{2} \mathrm{O}, 0.05 \mu \mathrm{l}$ of $2 \mathrm{U} / \mu \mathrm{l}$ Taq DNA ligase (New England Biolabs, Ipswich, MA, USA). The LDR was performed using 40 cycles of denaturation at $95^{\circ} \mathrm{C}$ for 2 min, annealing at $94{ }^{\circ} \mathrm{C}$ for $15 \mathrm{~s}$ and extension at $50^{\circ} \mathrm{C}$ for $25 \mathrm{~s}$. The LDR fluorescent product was analyzed using an ABI sequencer 377 (Applied Biosystems), and the results were analyzed with Genemapper Software (Applied Biosystems).

In addition, to test the validity of the assay, we made double positive controls (duplication of the same DNA samples) and negative controls (samples without DNA) in genotyping procedure. Quality-control analysis was also performed so that only SNPs and samples that had passed the $95 \%$ quality-control threshold were subjected to further statistical analysis.

\section{Statistical analysis}

All statistical data analyses were performed with SPSS version 17.0 Software (SPSS, Inc.). Association tests for genotype/allele frequency of each SNP were done using the two-tailed Pearson $\chi^{2}$-test. Compliance with HardyWeinberg equilibrium (HWE) was assessed using the HWE program (http://ihg.gsf.de/cgi-bin/hw/hwa1.pl) in the control population for each SNP. A linkage disequilibrium (LD) map used to define the haplotype blocks was constructed using Haploview Software (version 4.2; http://www.broadinstitute.org/haploview/haploview). The haplotype analysis was performed to compare distributions of haplotype frequencies of SNPs between cases and controls. Differences in the distributions of demographic characteristics variables, allele and genotype frequencies of four SNPs between the patients and controls were estimated by the $\chi^{2}$-test. The clinical and experimental indexes of different AITD phenotypes were compared by $t$-test. To examine whether each SNP independently contributes to susceptibility to AITD,

Published by Bioscientifica Ltd. 
Table 1 Clinical and demographic characteristic of AITD patients and controls

\begin{tabular}{l} 
Characteristics \\
\hline Gender \\
Male (\%) \\
Female (\%) \\
Age (mean \pm s.d.) \\
Onset of age \\
$\leq 18$ years \\
$\geq 19$ years \\
Thyroid size \\
Normal size (\%) \\
Degree I (\%) \\
Degree II (\%) \\
Degree III (\%) \\
Family history (\%) \\
Ophthalmopathy (\%) \\
Thyroid antibodies \\
TPOAb \\
TgAb
\end{tabular}

\begin{tabular}{c}
\hline GD patients $(n=701)$ \\
\hline $214(30.5)$ \\
$487(69.5)$ \\
$36.86 \pm 14.549$ \\
$33.98 \pm 14.344$ \\
$113(16.12)$ \\
$588(83.88)$ \\
$22(3.1)$ \\
$127(18.1)$ \\
$425(60.7)$ \\
$127(18.1)$ \\
$174(24.8)$ \\
$135(19.3)$ \\
$398(56.7)$ \\
$303(43.2)$
\end{tabular}

conditional logistic regression analysis was conducted. $P$ value $<0.05$ was taken as statically significant. Odds ratio (OR) and 95\% CI were calculated for the association between each genotype and AITD.

\section{Power calculation}

The power of the data was evaluated using the Power and Sample Size Calculation Software (Ps; version 3.0; http:// biostat.mc.vanderbilt.edu/twiki/bin/view/Main/PowerSampleSize) (Dupont \& Plummer 1998)). As the MAF of rs2723186 was the lowest among the four SNPs according to the Hapmap CHB data, we calculated the power of rs2323186. Considering the expected frequency of minor allele of rs2723186 in controls, the combined set of 760 GD patients and 938 controls gave a power of $94.1 \%$ with an $\mathrm{OR}=0.77$ at the $5 \%$ significance level.

\section{Results}

\section{Demographic and clinical characteristics of each group}

The demographic and clinical characteristics of all subjects are summarized in Table 1. Among GD patients, 69.5\% were females and the mean age was 36.86 years. Of the HT patients, $87.8 \%$ were females and the mean age was 35.09 years. In the control group, $67.1 \%$ individuals were females and the overall mean age was 38.61 years. There was no difference concerning the age and gender distribution between GD patients and controls. There was no difference in age distribution between HT patients

\begin{tabular}{|c|c|}
\hline HT patients $(n=360)$ & Controls $(n=938)$ \\
\hline $44(12.2)$ & 309 (32.9) \\
\hline $316(87.8)$ & $629(67.1)$ \\
\hline $35.09 \pm 13.786$ & $38.61 \pm 9.202$ \\
\hline $32.79 \pm 13.465$ & - \\
\hline $51(14.2)$ & - \\
\hline \multirow[t]{2}{*}{$309(85.8)$} & - \\
\hline & - \\
\hline $12(3.3)$ & - \\
\hline $59(16.4)$ & - \\
\hline $253(70.3)$ & - \\
\hline $36(10.0)$ & - \\
\hline \multicolumn{2}{|l|}{79 (21.9) } \\
\hline \multirow[t]{2}{*}{$9(2.5)$} & - \\
\hline & - \\
\hline $252(70.0)$ & - \\
\hline $226(62.8)$ & - \\
\hline
\end{tabular}

and controls, either. However, female HT patients outnumbered male HT ones by seven times.

\section{Association study of IL37 polymorphism with AITD}

The distribution of genotype frequencies for each SNP (rs3811046/rs3811047/rs2723176/rs2723186) was in Hardy-Weinberg equilibrium in both the patients and the controls (Table 2).

The genotypic and allelic distribution of four SNPs were analyzed in AITD, GD and HT patients and compared with those of controls. The results are summarized in Table 3. rs2723176 and rs2723186 polymorphisms appeared to be associated with AITD susceptibility. For rs2723176, the minor allele A frequency was significantly lower in both AITD and GD groups than in the control group and seemed to decrease the risks for AITD and GD (in AITD $13.3 \%$ vs $15.6 \%, P=0.044, \mathrm{OR}=0.832$, $95 \% \mathrm{CI}=0.696-0.995$; in GD, $12.5 \%$ vs $15.6 \%, P=0.014$, $\mathrm{OR}=0.774,95 \% \mathrm{CI}=0.631-0.949$ ). Similar results were also found for rs2723186. A significant decrease in the frequency of the minor A allele was observed in AITD and GD patient groups compared with that in the control

Table 2 Hardy-Weinberg $P$ value of the four SNPs

\begin{tabular}{|c|c|c|c|}
\hline SNP ID & Control & GD & HT \\
\hline rs3811046 G/T & 0.441 & 0.426 & 0.331 \\
\hline $\mathrm{rs} 3811047 \mathrm{~A} / \mathrm{G}$ & 0.759 & 0.735 & 0.467 \\
\hline rs2723176 C/A & 0.585 & 0.244 & 0.585 \\
\hline rs2723186 A/G & 0.228 & 0.236 & 0.911 \\
\hline
\end{tabular}

Published by Bioscientifica Ltd. 
Table 3 Genotype and allele distribution of four SNPs in IL37 gene in AITDs patients, GD patients, HT patients and controls

\begin{tabular}{|c|c|c|c|c|c|c|c|c|}
\hline \multirow[b]{2}{*}{ SNP } & \multicolumn{3}{|c|}{ Allele } & \multicolumn{2}{|l|}{$\boldsymbol{P}_{\mathbf{a}}$} & \multicolumn{2}{|l|}{$\boldsymbol{P}_{\mathrm{b}}$} & \multirow{2}{*}{$\frac{\boldsymbol{P}_{\mathbf{c}}}{(\mathrm{OR}, 95 \% \mathrm{Cl})}$} \\
\hline & Genotype & Controls & AITD & $(\mathrm{OR}, 95 \% \mathrm{Cl})$ & GD & $(\mathrm{OR}, 95 \% \mathrm{Cl})$ & HT & \\
\hline \multirow[t]{5}{*}{ rs 3811046} & GG & $36(4.1)$ & $40(4.0)$ & & $21(3.2)$ & & $19(5.5)$ & \\
\hline & GT & $266(30.2)$ & $288(28.6)$ & 0.712 & $177(26.8)$ & 0.182 & $111(31.9)$ & 0.444 \\
\hline & $\mathrm{TT}$ & $578(65.7)$ & $680(67.4)$ & & $462(70.0)$ & & $218(62.6)$ & \\
\hline & $\mathrm{G}$ & $338(19.2)$ & $368(18.3)$ & 0.455 & $219(16.6)$ & 0.062 & $149(21.4)$ & 0.217 \\
\hline & $\mathrm{T}$ & $1422(80.8)$ & $1648(81.7)$ & & 1101 (83.4) & & $547(78.6)$ & \\
\hline \multirow[t]{5}{*}{ rs 3811047} & AA & $38(4.2)$ & $42(4.0)$ & & $22(3.2)$ & & $20(5.6)$ & \\
\hline & AG & $288(31.8)$ & $313(30.0)$ & 0.677 & $195(28.3)$ & 0.153 & $118(33.3)$ & 0.417 \\
\hline & GG & $581(64.0)$ & $688(66.0)$ & & $472(68.5)$ & & $216(61.1)$ & \\
\hline & A & $364(20.0)$ & $397(19.0)$ & 0.416 & $239(17.3)$ & 0.052 & $158(22.3)$ & 0.210 \\
\hline & $\mathrm{G}$ & $1450(80.0)$ & $1689(81.0)$ & & $1139(82.7)$ & & $550(77.7)$ & \\
\hline \multirow[t]{5}{*}{ rs 2723176} & $A A$ & $20(2.2)$ & $21(2.0)$ & & $14(2.0)$ & & $7(2.0)$ & \\
\hline & $A C$ & $245(26.7)$ & $235(22.6)$ & 0.089 & $143(20.9)$ & 0.023 & $92(25.8)$ & 0.903 \\
\hline & $\mathrm{CC}$ & $651(71.1)$ & 786 (75.4) & & $528(77.1)$ & & $258(72.2)$ & \\
\hline & $A$ & $285(15.6)$ & $277(13.3)$ & 0.044 & $171(12.5)$ & 0.014 & $106(14.8)$ & 0.655 \\
\hline & $\mathrm{C}$ & $1547(84.4)$ & $1807(86.7)$ & $0.832(0.696-0.995)$ & 1199 (87.5) & $0.774(0.631-0.949)$ & $608(85.2)$ & \\
\hline \multirow[t]{5}{*}{ rs2723186 } & $A A$ & $19(2.1)$ & $23(2.2)$ & & $15(2.2)$ & & $8(2.3)$ & \\
\hline & AG & $259(28.2)$ & $238(23.2)$ & 0.044 & $147(21.6)$ & 0.012 & $91(26.3)$ & 0.793 \\
\hline & GG & $643(69.7)$ & 766 (74.6) & & $519(76.2)$ & & $247(71.4)$ & \\
\hline & $A$ & $297(16.1)$ & $284(13.8)$ & & $177(13.0)$ & 0.014 & $107(15.5)$ & 0.685 \\
\hline & G & 1545 (83.9) & $1770(86.2)$ & $0.835(0.700-0.996)$ & $1185(87.0)$ & $0.777(0.636-0.950)$ & $585(84.5)$ & \\
\hline
\end{tabular}

$P_{\mathrm{a}}$ value, AITD vs controls; $P_{\mathrm{b}}$ value, GD vs controls; $P_{\mathrm{c}}$ value, HT vs controls; OR, odd ratio.

group (in AITD, $13.8 \%$ vs $16.1 \%, P=0.044, \mathrm{OR}=0.835$, $95 \% \mathrm{CI}=0.700-0.996$; in $\mathrm{GD}, 13.0 \%$ vs $16.1 \%, P=0.014$, $\mathrm{OR}=0.777,95 \% \mathrm{CI}=0.636-0.950)$. Comparisons of the genotype frequencies also showed obvious differences in genotype distributions between GD patients and healthy controls (rs2723176, $P=0.023$; rs2723186, $P=0.012$ ). However, we could not find significant differences in the genotype or allele frequencies of two SNPs (rs3811046 and rs3811047) between AITD patients, GD patients, HT patients and healthy controls.

In further analysis for the association of four SNPs with susceptibility to AITD in female and male groups (Table 4), the minor G allele of rs3811046 and the minor A allele of rs3811047 were significantly associated with a decrease in GD risk in female group $(P=0.030, \mathrm{OR}=0.777$, 95\% CI $=0.618-0.976 ; P=0.023, \quad \mathrm{OR}=0.774,95 \% \mathrm{CI}=$ $0.620-0.966$ respectively). The frequencies of rs2723186 genotypes in female GD patients differed significantly from those in the controls $(P=0.023)$; the A allele frequency was significantly lower than that of healthy controls $(P=0.029, \mathrm{OR}=0.761,95 \% \mathrm{CI}=0.596-0.973)$. It was found that the frequency of A allele of rs2723176 was lower in GD female group but without statistical significance $(P=0.053)$. However, all four SNPs of IL37 gene did not reveal any significant associations with HT in female or male patients.
To evaluate the independent genetic effect of each SNP on the susceptibility to AITD, conditional logistic regression analysis was performed. As shown in Table 5, the association of rs2723186 remained significant after adjustment for rs2723176 SNP genotypes. Adjusted $P$ values ( $P$ adjusted) for rs2723186 under the dominant model were 0.041 and 0.036 in AITD and GD after adjustment for rs2723176. The results suggest that rs2723186 SNP is independently associated with GD.

\section{Analysis of haplotypic association}

The four SNPs of IL37 gene were tightly linked to each other; a strong linkage disequilibrium was observed among rs3811047, rs2723176 and rs2723816 in controls using the Haploview 4.2, especially these three SNPs that were in the same LD block (Table 6). To further identify whether haplotypes of the IL37 gene were correlated with AITD, we created an LD map (Fig. 1) and analyzed haplotype frequency differences between AITD patients and controls (Table 7). Haplotype analysis showed that three haplotypes, GCG, AAA and ACG, were detected in the IL37 gene. The frequency of haplotype GCG with GD patients was significantly higher than that in their control group $(P=0.034)$, the GCG haplotype conferred a significant increased risk for $\mathrm{GD}$ as a whole $(\mathrm{OR}=1.213$,

Published by Bioscientifica Ltd. 
Table 4 Analysis of genotype and allele distribution of four SNPs in GD patients, HT patients and controls after gender stratification

\begin{tabular}{|c|c|c|c|c|c|c|c|c|c|c|c|}
\hline \multirow[b]{3}{*}{ SNP } & \multicolumn{4}{|c|}{ Genotype } & \multicolumn{5}{|c|}{$\boldsymbol{P}_{\mathrm{a}}$} & \multirow{2}{*}{\multicolumn{2}{|c|}{$\frac{\boldsymbol{P}_{\mathbf{b}}}{\mathrm{OR}(95 \% \mathrm{Cl})}$}} \\
\hline & \multirow[t]{2}{*}{ Allele } & \multicolumn{2}{|c|}{ Controls } & \multicolumn{2}{|c|}{ GD } & \multicolumn{2}{|c|}{$\mathrm{HT}$} & \multicolumn{2}{|r|}{ OR $(95 \% \mathrm{Cl})$} & & \\
\hline & & $M$ & $\mathrm{~F}$ & $M$ & $\mathrm{~F}$ & $\mathrm{M}$ & $\mathrm{F}$ & $\mathrm{M}$ & $\mathrm{F}$ & $\mathrm{M}$ & $\mathrm{F}$ \\
\hline \multirow[t]{5}{*}{ rs3811046 } & GG & $9(3.1)$ & $27(4.6)$ & $7(3.5)$ & $14(3.0)$ & $2(4.7)$ & $17(5.6)$ & & & & \\
\hline & GT & 92 (31.3) & $174(29.7)$ & $59(29.8)$ & $118(25.5)$ & $11(25.6)$ & $100(32.8)$ & 0.911 & 0.106 & 0.675 & 0.468 \\
\hline & $\mathrm{TT}$ & $193(65.6)$ & $385(65.7)$ & $132(66.7)$ & $330(71.5)$ & $30(69.7)$ & $188(61.6)$ & & & & \\
\hline & G & $110(18.7)$ & $228(19.5)$ & $73(18.4)$ & $146(15.8)$ & $15(17.4)$ & $134(22.0)$ & 0.914 & 0.030 & 0.778 & 0.211 \\
\hline & $\mathrm{T}$ & $478(81.3)$ & $944(80.5)$ & $323(81.6)$ & $778(84.2)$ & $71(82.6)$ & $476(78.0)$ & & $0.777(0.618-0.976)$ & & \\
\hline \multirow[t]{5}{*}{ rs3811047 } & AA & $9(3.0)$ & $29(4.8)$ & $7(3.3)$ & $15(3.1)$ & $2(4.7)$ & $18(5.8)$ & & & & \\
\hline & AG & $101(33.4)$ & $187(30.9)$ & $68(32.3)$ & $127(26.6)$ & $12(27.9)$ & $106(34.1)$ & 0.944 & 0.083 & 0.681 & 0.447 \\
\hline & GG & $192(63.6)$ & $389(64.3)$ & $136(64.4)$ & $336(70.3)$ & $29(67.4)$ & $187(60.1)$ & & & & \\
\hline & $A$ & $119(19.7)$ & $245(20.2)$ & $82(19.4)$ & $157(16.4)$ & $14(16.7)$ & $142(22.8)$ & 0.914 & 0.023 & 0.509 & 0.200 \\
\hline & G & $485(80.3)$ & $965(79.8)$ & $340(80.6)$ & $799(83.6)$ & $70(83.3)$ & $480(77.2)$ & & $0.774(0.620-0.966)$ & & \\
\hline \multirow[t]{5}{*}{ rs2723176 } & AA & $5(1.6)$ & $15(2.5)$ & $2(0.9)$ & $12(2.5)$ & $0(0.0)$ & $7(2.2)$ & & & & \\
\hline & $A C$ & $89(29.2)$ & $156(25.5)$ & $50(23.8)$ & $93(19.6)$ & $11(25.0)$ & $81(25.9)$ & 0.302 & 0.068 & 0.565 & 0.974 \\
\hline & $\mathrm{CC}$ & $211(69.2)$ & $440(72.0)$ & $158(75.3)$ & 370 (77.9) & $33(75.0)$ & 225 (71.9) & & & & \\
\hline & A & $99(16.2)$ & $186(15.2)$ & 54 (12.9) & $117(12.3)$ & $11(12.5)$ & $95(15.2)$ & 0.135 & 0.053 & 0.369 & 0.98 \\
\hline & $\mathrm{C}$ & $511(83.8)$ & $1036(84.8)$ & $366(87.1)$ & $833(87.7)$ & $77(87.5)$ & $531(84.8)$ & & & & \\
\hline \multirow[t]{5}{*}{ rs2723186 } & AA & $5(1.6)$ & $14(2.3)$ & $3(1.4)$ & $12(2.5)$ & $0(0.0)$ & $8(2.6)$ & & & & \\
\hline & AG & $91(29.6)$ & $168(27.4)$ & $52(24.8)$ & $95(20.2)$ & $10(23.3)$ & $81(26.7)$ & 0.458 & 0.023 & 0.454 & 0.932 \\
\hline & GG & $211(68.8)$ & $432(70.3)$ & $155(73.8)$ & $364(77.3)$ & $33(76.7)$ & $214(70.7)$ & & & & \\
\hline & $A$ & $101(16.4)$ & $196(16.0)$ & $58(13.8)$ & $119(12.6)$ & $10(11.6)$ & $97(16.0)$ & 0.248 & 0.029 & 0.252 & 0.98 \\
\hline & G & $513(83.6)$ & $1032(84.0)$ & $362(86.2)$ & $823(87.4)$ & $76(88.4)$ & $509(84.0)$ & & $0.761(0.596-0.973)$ & & \\
\hline
\end{tabular}

M, male; F, female; $P_{\mathrm{a}}$ value, GD vs controls; $P_{\mathrm{b}}$ value, HT vs controls; OR, odd ratio.

95\% CI=1.015-1.450); in females, the GCG haplotype was also associated with an increase risk of $G D(\mathrm{OR}=$ $1.320,95 \% \mathrm{CI}=1.061-1.642, P=0.012)$. The frequency of haplotype ACG was significantly higher in HT patients than in the controls $(P=0.013)$; the ACG haplotype was associated with an obviously increased risk of HT $(\mathrm{OR}=$ 1.567, 95\% CI=1.096-2.241, $P=0.013$ ). In males, the frequencies of the ACG haplotype in AITD patients and GD patients were significantly higher than those in control groups $(P=0.037,0.036)$, indicating that ACG was a risk haplotype for GD $(\mathrm{OR}=1.820,95 \% \mathrm{CI}=1.033-$ 3.205), but not for HT. In contrast, the frequencies of haplotype AAA were significantly lower in AITD and GD patient groups than those in their control groups $(P=0.037,0.009)$, suggesting that the AAA haplotype showed a protective role for AITD susceptibility as a whole $(\mathrm{OR}=0.826,95 \% \mathrm{CI}=0.691-0.989)$, especially for $\mathrm{GD}$ $(\mathrm{OR}=0.760,95 \% \mathrm{CI}=0.619-0.934)$. In females, the AAA haplotype was also associated with a decreased risk for GD (OR=0.765, 95\% CI=0.596-0.983).

Table 5 Interaction analysis of gene-gene in AITD, GD and HT patients by logistic regression ${ }^{\text {a }}$.

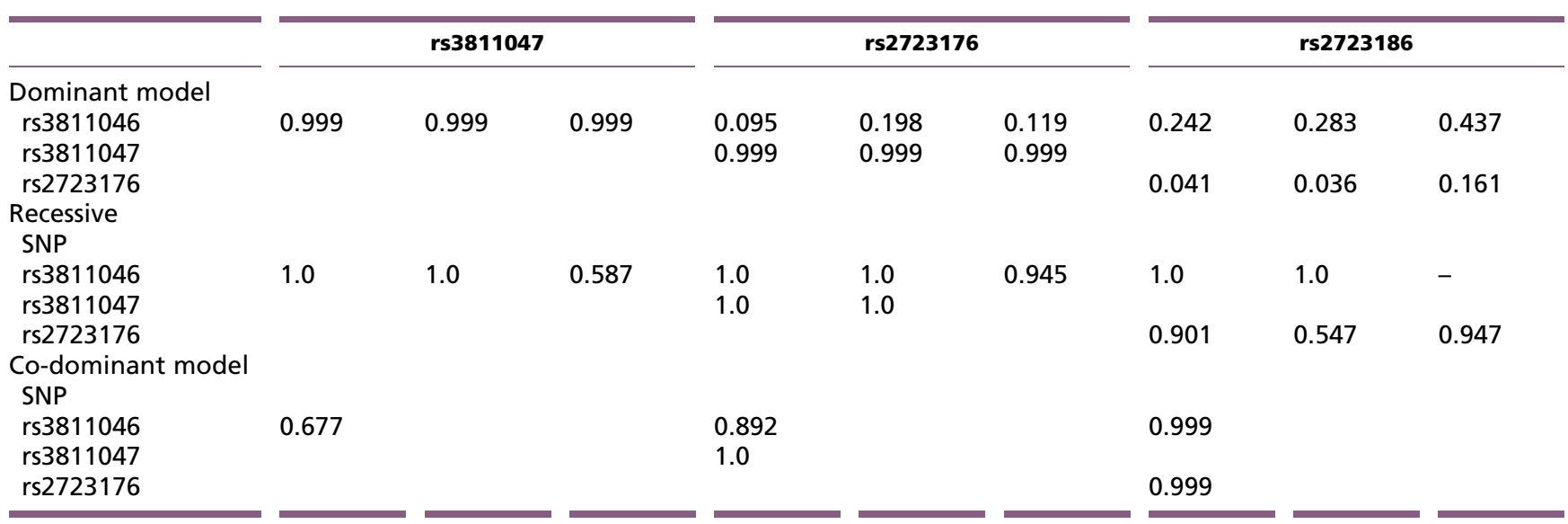

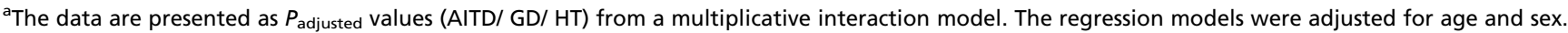

http://jme.endocrinology-journals.org DOI: 10.1530/JME-15-0144
(C) 2015 Society for Endocrinology Printed in Great Britain
Published by Bioscientifica Ltd. 
Table 6 Linkage disequilibrium in controls

\begin{tabular}{|c|c|c|c|}
\hline L1 & $\mathbf{L 2}$ & $\mathbf{D}^{\prime}$ & $r^{2}$ \\
\hline rs3811047 & rs2723176 & 1.0 & 0.721 \\
\hline rs3811047 & rs2723186 & 0.95 & 0.684 \\
\hline rs2723176 & rs2723186 & 1.0 & 0.952 \\
\hline
\end{tabular}

\section{Genotype and clinical phenotype correlations}

The genotypes and alleles of the selected four SNPs were tested for interactions with such risk factors of AITD as cigarette smoking and family history, but without any notable effect (data not shown). Clinical parameters of AITD, including age of onset ( $\leq 18$ years or $\geq 19$ years), GD with or without ophthalmopathy, intractable GD or GD in remission and HT patients with thyroid dysfunction (hypothyroidism or normal), were not associated with genotype or allele variants of the four tested SNPs either (data not shown).

\section{Discussion}

To the best of our knowledge, the present study was the first demonstration that the IL37 polymorphism is associated with susceptibility to AITD. The results of our study showed that carriers of the minor A allele of rs2723176 and rs2723186 were associated with a significantly decreased risk of GD; Moreover, the minor $\mathrm{G}$ allele of rs3811046 and the minor A allele of rs3811047 also significantly reduced the risk of GD in female patients. This suggests that the minor A allele of rs2723176/rs2723186/ rs3811047and the minor G allele of rs3811046 were the protective factors for GD. Our study contributes to the understanding of individual susceptibility to AITD when it is a carrier of protective alleles of IL37, highlighting the relevance of polymorphisms in inflammation-related gene as molecular marker for AITD.

IL37 (IL1F7) has five splice variants (IL37a-e) and is the only IL1 family member that is not found in mice (Boraschi et al. 2011). Recent studies revealed that IL37 has a strong anti-inflammatory effect during an innate immune response as well as in the development of inflammation (Nold et al. 2010, Banchereau et al. 2012). Published literature suggests that IL37 is expressed in various normal tissues, tumors (Pan et al. 2001, Kumar et al. 2002) and by human peripheral blood mononuclear cells under inflammatory conditions, such as systemic lupus erythematosus (SLE) (Song et al. 2013), Behcet's disease (BD) (Ye et al. 2014) and acute coronary syndrome (Ji et al. 2014). Genetic variants in inflammation-related cytokine genes may have the potential to alter the regulation of transcript production or function. Considering the role of the inflammation-related cytokines in the pathogenesis of the autoimmune response involved in AITD, a change in the function or the quantity of a particular cytokine may lead to the initiation or inhibition of the inflammatory process. The importance of IL37 in the immunological response is well established in existing literature. Thus, SNPs in the cytokine may have a role in the susceptibility to HT and GD in which the immune response is a major feature.

SNP rs3811046 (G/T transition) and rs3811047 (A/G transition) were located in exon 2 (amino acid position 31, 42 respectively). Both of the SNPs were missense mutations resulting in different amino acid codons (rs3811046: Gly/Val; rs3811047:Thr/Ala). Polymorphism of rs3811047 has been implicated in susceptibility to RA (Pei et al. 2012, Shi et al. 2013 33), AS (Pan et al. 2010), and gastric cardiac adenocarcinoma (Wang et al.2014). A study which recruited subjects (276 controls and 276 RA patients) from central plains of Chinese Han population indicated no significant correlation between RA
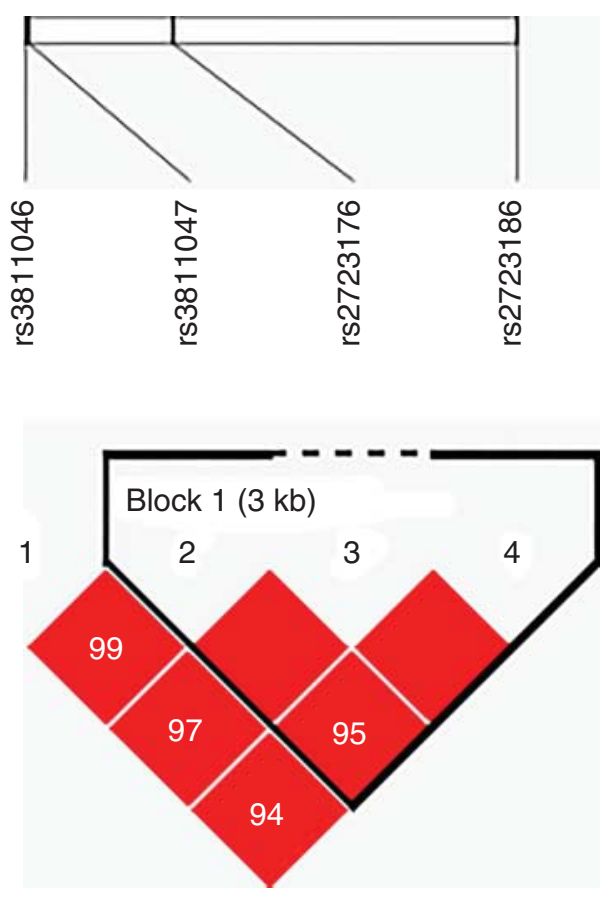

Figure 1

Four tag SNPs on the LD map of IL37 gene. Patterns of linkage disequilibrium (LD) between the IL37 single nucleotide polymorphisms (SNPs). The strength of LD measured by $\mathrm{D}^{\prime}$ multiplying 100 is displayed in the red diamonds. A red cell without number indicates a complete LD. A Larger number in the cell indicates a higher degree of LD. A full colour version of this figure is available at http://dx.doi.org/10.1530/JME-15-0144.

Published by Bioscientifica Ltd. 


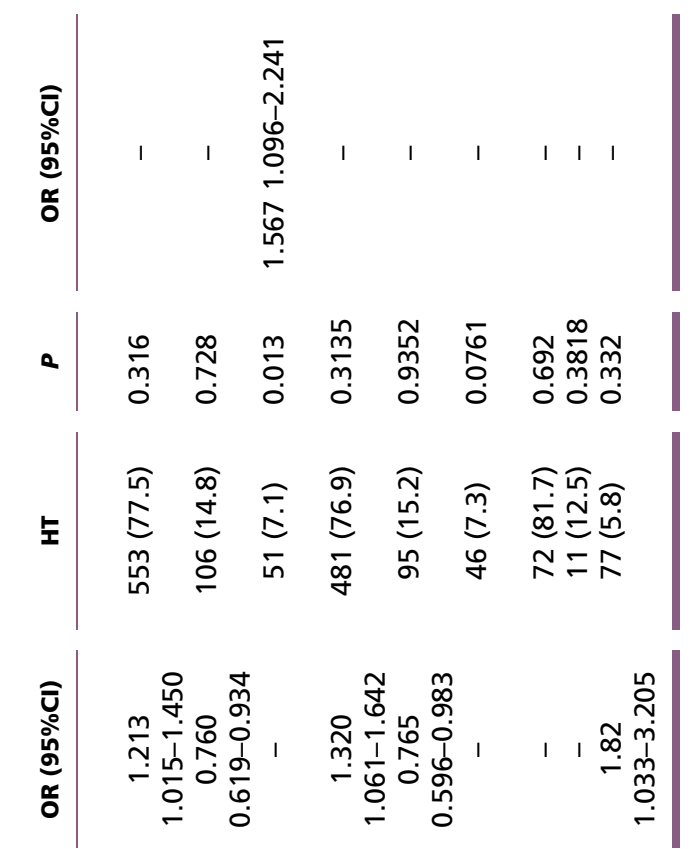

$a$

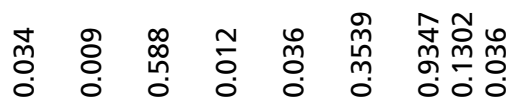

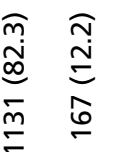

$$
\text { ๖ำ }
$$

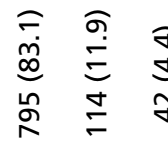

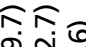

จำ

$\stackrel{\infty}{m}$ ஸे
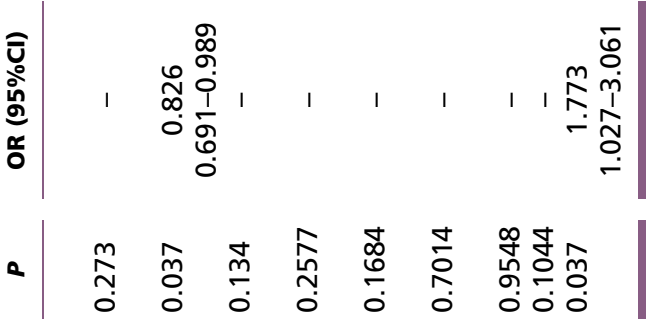

$$
\text { ำ }
$$

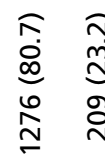

$$
\text { อุ }
$$$$
\text { ำกิก }
$$$$
\text { 宅气 }
$$
웡

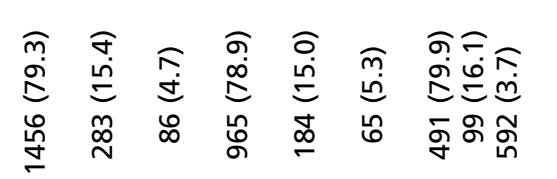

susceptibility and rs3811047 SNP in IL37 gene; however, it also showed that RA patients with A allele were significantly better than those without A allele in joint swelling index, rest pain, HAQ scoring and blood sedimentation (Shi et al. 2013). Another Chinese group had synchronously reported no statistically significant differences in the genetic polymorphism (genotypes and allele frequencies) of rs3811047 in the RA patients compared with controls (Pei et al. 2012). They also found that those carrying A allele had less severe disease activity, suggesting that the A allele of rs3811047 may have a protective effect on RA. Wang et al. detected the allelic distribution of rs3811047 G>A polymorphism in 243 gastric cardiac adenocarcinoma cases and 476 controls, and observed that the rs3811047 G > A polymorphism was significantly associated with a decreased risk of gastric cardiac adenocarcinoma either in the single locus analysis or the recessive genetic model (Wang et al. 2014). In agreement with existing studies, our study reports associations of the minor A allele of rs3811047 and the minor G allele of rs3811046 with protective effects from developing GD in female patients but not HT patients in a large case-control Chinese Han population.

SNP rs2723176 and rs2723186 were both in the intron region. As far as we know, there is hardly any study on the polymorphism of the above two SNPs. We analyzed for the first time the associations of rs2723176/rs2723186 polymorphisms with susceptibility to AITD. Our results demonstrated that the minor A allele of rs2723176/ rs2723186 may be a protective factor for GD, can significantly decrease the risk of $\mathrm{GD}(\mathrm{OR}=0.774,0.777$ respectively), but does not associate with HT. After gender stratification, only the A allele of rs 2723186 is significantly associated with a decreased risk in female GD patients $(\mathrm{OR}=0.761)$.

Haplotype analysis is likely to continue to play an important role in genetic epidemiology studies (Schaid 2004) because it effectively captures both the joint marker correlations and the evolutionary history. Therefore, we carried out the haplotype analysis of the tested four SNPs between AITD patients and controls. The rate of haplotype GCG was significantly higher in GD, conferring a significant degree of risk for GD as a whole and in female GD patients. The rate of haplotype ACG was significantly higher in HT, suggesting an obviously increased risk of HT. In contrast, the rate of haplotype AAA was significantly lower in GD, showing a protective role for GD susceptibility. These results are consistent with those of the allele analysis of the four SNPs.

Published by Bioscientifica Ltd. 
It is worthwhile to point out that several limitations exist in our study. Firstly, because of limited SNPs included in our study, future studies using the high-throughput analytic methods available (i.e., gene sequencing) may help clarify our findings and provide further insights into the role of IL37 in AITD. In addition, the mechanisms by which four SNPs exert their roles are not comprehensively understood. Therefore, further studies are required to determine the functional variants of IL37 SNPs in AITD. Finally, findings from this study cannot be extrapolated to other ethnic groups. The relevance of IL37 gene risk variants to additional autoimmune diseases, autoimmune disease susceptibility in other populations and specific disease sub-phenotypes and outcomes needs to be addressed in large, well-characterized cohorts.

\section{Conclusion}

We were the first to evaluate the correlation of polymorphisms in IL37 gene with the susceptibility to AITD. Our study suggests that the minor A allele of rs3811047/rs2723176/rs2723186 and the minor $G$ allele of rs3811046 have a protective influence on GD susceptibility.

\section{Declaration of interest}

The authors declare that there is no conflict of interest that could be perceived as prejudicing the impartiality of the research reported.

\section{Funding}

This project was supported by grants from the National Natural Science Foundation of China $(81270871,81471004)$ and the Key Disciplines Development of Shanghai Jinshan District (No. 2012-225).

\section{Author contribution statement}

N Y, S M, Q Q, X W, and R S carried out the work. J X, W J, L S, Q Y and $\mathrm{Y} J$ helped with the collection of specimens. $N$ Y conducted the data analysis and wrote the manuscript and $J Z$ coordinated the research team. All authors have read and approved the final manuscript.

\section{Acknowledgements}

The authors would like to thank all of the people who participated in the studies.

\section{References}

Antonelli A, Ferrari SM, Corrado A, Di Domenicantonio A \& Fallahi P 2015 Autoimmune thyroid disorders. Autoimmunity Reviews 14 174-180. (doi:10.1016/j.autrev.2014.10.016)
Anvari M, Khalilzadeh O, Esteghamati A, Momen-Heravi F, Mahmoudi M, Esfahani SA, Rashidi A \& Amirzargar A 2010 Graves' disease and gene polymorphism of TNF- $\alpha$, IL-2, IL-6, IL-12, and IFN- $\gamma$. Endocrine 37 344-348. (doi:10.1007/s12020-010-9311-y)

Banchereau J, Pascual V \& O'Garra A 2012 From IL-2 to IL37: the expanding spectrum of anti-inflammatory cytokines. Nature Immunology 13 925-931. (doi:10.1038/ni.2406)

Boraschi D, Lucchesi D, Hainzl S, Leitner M, Maier E, Mangelberger D, Oostingh GJ, Pfaller T, Pixner C, Posselt G et al. 2011 IL37: a new anti-inflammatory cytokine of the IL-1 family. European Cytokine Network 22 127-147. (doi:10.1684/ecn.2011.0288)

Chen RH, Chen WC, Wang TY, Tsai CH \& Tsai FJ 2005 Lack of association between pro-inflammatory cytokine (IL-6, IL-8 and TNF- $\alpha$ ) gene polymorphisms and Graves' disease. International Journal of Immunogenetics 32 343-347. (doi:10.1111/j.1744-313X.2005.00536.x)

Dominici R, Cattaneo M, Malferrari G, Archi D, Mariani C, Grimaldi LM \& Biunno I 2002 Cloning and functional analysis of the allelic polymorphism in the transcription regulatory region of interleukin- $1 \alpha$. Immunogenetics 54 82-86. (doi:10.1007/s00251-002-0445-9)

Dupont WD \& Plummer WD Jr 1998 Power and sample size calculations for studies involving linear regression. Controlled Clinical Trials 19 589-601. (doi:10.1016/S0197-2456(98)00037-3)

Ganesh BB, Bhattacharya P, Gopisetty A \& Prabhakar BS 2011 Role of cytokines in the pathogenesis and suppression of thyroid autoimmunity. Journal of Interferon \& Cytokine Research 31 721-731. (doi:10.1089/jir.2011.0049)

Ji Q, Zeng Q, Huang Y, Shi Y, Lin Y, Lu Z, Meng K, Wu B, Yu K, Chai M et al. 2014 Elevated plasma IL37, IL-18, and IL-18BP concentrations in patients with acute coronary syndrome. Mediators of Inflammation article 165742. (doi:10.1155/2014/165742)

Kumar S, Hanning CR, Brigham-Burke MR, Rieman DJ, Lehr R, Khandekar S, Kirkpatrick RB, Scott GF, Lee JC, Lynch FJ et al. 2002 Interleukin-1F7B (IL-1H4/IL-1F7) is processed by caspase- 1 and mature IL-1F7B binds to the IL-18 receptor but does not induce IFN- $\gamma$ production. Cytokine $\mathbf{1 8}$ 61-71. (doi:10.1006/cyto.2002.0873)

Liu N, Li X, Liu C, Zhao Y, Cui B \& Ning G 2010 The association of interleukin- $1 \alpha$ and interleukin-1 $\beta$ polymorphisms with the risk of Graves' disease in a case-control study and meta-analysis. Human Immunology 71 397-401. (doi:10.1016/j.humimm.2010.01.023)

McNamee EN, Masterson JC, Jedlicka P, McManus M, Grenz A, Collins CB, Nold MF, Nold-Petry C, Bufler P, Dinarello CA et al. 2011 Interleukin 37 expression protects mice from colitis. PNAS 108 16711-16716. (doi:10.1073/pnas.1111982108)

Miceli-Richard C, Zouali H, Said-Nahal R, Lesage S, Merlin F, De Toma C, Blanche H, Sahbatou M, Dougados M, Thomas G et al. 2004 Significant linkage to spondyloarthropathy on 9q31-34. Human Molecular Genetics 13 1641-1648. (doi:10.1093/hmg/ddh179)

Morshed SA, Latif R \& Davies TF 2012 Delineating the autoimmune mechanisms in Graves' disease. Immunologic Research 54 191-203. (doi:10.1007/s12026-012-8312-8)

Nicklin MJ, Barton JL, Nguyen M, FitzGerald MG, Duff GW \& Kornman K 2002 A sequence-based map of the nine genes of the human interleukin-1 cluster. Genomics 79 718-725. (doi:10.1006/geno. 2002.6751)

Nold MF, Nold-Petry CA, Zepp JA, Palmer BE, Bufler P \& Dinarello CA 2010 IL37 is a fundamental inhibitor of innate immunity. Nature Immunology 11 1014-1022. (doi:10.1038/ni.1944)

Pan G, Risser P, Mao W, Baldwin DT, Zhong AW, Filvaroff E, Yansura D, Lewis L, Eigenbrot C, Henzel WJ et al. $2001 \mathrm{IL}-1 \mathrm{H}$, an interleukin 1-related protein that binds IL-18 receptor/IL-1Rrp. Cytokine 1 1-7. (doi:10.1006/cyto.2000.0799)

Pan F, Liao F, Xia G, Ge R, Mei Y, Tang X, Pan H, Ye D, Zou Y, Xu S et al. 2010 Association of IL-1F7 gene with susceptibility to human leukocyte antigen-b27 positive ankylosing spondylitis in Han Chinese population. Clinica Chimica Acta $\mathbf{4 1 1}$ 124-126. (doi:10.1016/j.cca.2009. 10.014)

Published by Bioscientifica Ltc. 
Pei B, Xu S, Liu T, Pan F, Xu J \& Ding C 2012 Associations of the IL-1F7 gene polymorphisms with rheumatoid arthritis in Han Chinese population. International Journal of Immunogenetics 40 199-203. (doi:10.1111/ iji.12007)

Prasad KN, Nyati KK, Verma A, Rizwan A \& Paliwal VK 2010 Tumor necrosis factor- $\alpha$ polymorphisms and expression in Guillain-Barré syndrome. Human Immunology 71 905-910. (doi:10.1016/j.humimm.2010.06.013)

Sakai N, Van Sweringen HL, Belizaire RM, Quillin RC, Schuster R, Blanchard J, Burns JM, Tevar AD, Edwards MJ \& Lentsch AB 2012 Interleukin-37 reduces liver inflammatory injury via effects on hepatocytes and non-parenchymal cells. Journal of Gastroenterology and Hepatology 27 1609-1616. (doi:10.1111/j.1440-1746.2012.07187.x)

Schaid DJ 2004 Evaluating associations of haplotypes with traits. Genetic Epidemiology 27 348-364. (doi:10.1002/gepi.20037)

Shi LP, He Y \& Liu ZD 2013 Correlation between single nucleotide polymorphism of rs3811047 in IL-1F7 gene and rheumatoid arthritis susceptibility among Han population in central plains of China. Asian Pacific Journal of Tropical Medicine 6 73-75. (doi:10.1016/ S1995-7645(12)60204-1)

Shiau MY, Huang CN, Yang TP, Hwang YC, Tsai KJ, Chi CJ \& Chang YH 2006 Cytokine promoter polymorphisms in Taiwanese patients with Graves' disease. Clinical Biochemistry 40 213-217. (doi:10.1016/ j.clinbiochem.2006.11.009)

Song L, Qiu F, Fan Y, Ding F, Liu H, Shu Q, Liu W \& Li X 2013 Glucocorticoid regulates interleukin-37 in systemic lupus erythematosus. Journal of Clinical Immunology 33 111-117. (doi:10.1007/ s10875-012-9791-z)

Timms AE, Crane AM, Sims AM, Cordell HJ, Bradbury LA, Abbott A, Coyne MR, Beynon O, Herzberg I, Duff GW et al. 2004 The interleukin 1 gene cluster contains a major susceptibility locus for ankylosing spondylitis. American Journal of Human Genetics 75 587-595. (doi:10.1086/424695)
Tomer Y 2010 Genetic susceptibility to autoimmune thyroid disease: past, present, and future. Thyroid 20 715-725. (doi:10.1089/thy.2010.1644)

Tomer Y \& Huber A 2009 The etiology of autoimmune thyroid disease: a story of genes and environment. Journal of Autoimmunity 32 231-239. (doi:10.1016/j.jaut.2009.02.007)

Wang X, Yin J, Zheng L, Wang L, Shi Y, Tang W, Ding G, Liu C, Liu R, Chen S et al. 2014 The variant interleukin 1f7 rs3811047 G > A was associated with a decreased risk of gastric cardiac adenocarcinoma in a Chinese Han population. Tumour Biology 35 3509-3515. (doi:10.1007/ s13277-013-1463-y)

Wen AQ, Wang J, Feng K, Zhu PF, Wang ZG \& Jiang JX 2006 Effects of haplotypes in the interleukin $1 \beta$ promoter on lipopolysaccharideinduced interleukin $1 \beta$ expression. Shock 26 25-30. (doi:10.1097/01. shk.0000223125.56888.c7)

Yan N, Yu YL, Yang J, Qin Q, Zhu YF, Wang X, Song RH \& Zhang JA 2012 Association of interleukin-17A and -17F gene single-nucleotide polymorphisms with autoimmune thyroid diseases. Autoimmunity $\mathbf{4 5}$ 533-539. (doi:10.3109/08916934.2012.702814)

Yan N, Meng S, Zhou J, Xu J, Muhali FS, Jiang W, Shi L, Shi X \& Zhang J 2014 Association between STAT4 Gene Polymorphisms and Autoimmune Thyroid Diseases in a Chinese Population. International Journal of Molecular Sciences 15 12280-12293. (doi:10.3390/ ijms150712280)

Ye Z, Wang C, Kijlstra A, Zhou X \& Yang P 2014 A Possible Role for Interleukin 37 in the Pathogenesis of Behcet's Disease. Current Molecular Medicine 14 535-542. (doi:10.2174/1566524014666140414210831)

Zhang G, Luo J, Bruckel J, Weisman MA, Schumacher HR, Khan MA, Inman RD, Mahowald M, Maksymowych WP, Martin TM et al. 2004 Genetic studies in familial ankylosing spondylitis susceptibility. Arthritis \& Rheumatology 50 2246-2254. (doi:10.1002/art.20308)

Received in final form 17 August 2015

Accepted 11 September 2015

Accepted Preprint published online 15 September 2015
(C) 2015 Society for Endocrinology Printed in Great Britain 